\title{
Evolving Tiles for Automated Self-Assembly Design
}

\author{
Germán Terrazas, Marian Gheorghe, Graham Kendall, Natalio Krasnogor
}

\begin{abstract}
Self-assembly is a distributed, asynchronous mechanism that is pervasive across natural systems where hierarchical complex structures are built from the bottom-up. The lack of a centralised master plan, no external intervention, and preprogrammed interactions among entities are within its most relevant and technologically appealing properties. This paper tackles the self-assembly Wang tiles designability problem by means of artificial evolution. This research is centred in the use of tiles that are extended with rotation and probabilistic motion, and an evolutionary algorithm using the Morphological Image Analyses method as a fitness function. The obtained results support this approach as a successful engineering mechanism for the computer-aided design of self-assembled patterns.
\end{abstract}

\section{INTRODUCTION}

Self-assembly systems are ubiquitous in nature and can be found at all scales. At the microscopic level, protein folding, lipids membranes, nucleic acid structures and other complex cell entities are well-known biological examples of self-assembly. Molecular crystals such as the crystalline form of table sugar and rock candy or colloids such as milk, butter and asphalt are everyday cases of self-assembly where molecules are kept together by interactive forces. On the other hand, swarms, school fish and solar systems are macroscopic dynamic selfassembly systems. Furthermore, information systems are yet another field where self-assembly appears as it plays an important role in novel software development techniques [1].

Self-assembly is a distributed process that creates structures from scratch through the statistical exploration of components' aggregates without human intervention. In general, self-assembly components are autonomous, have no pre-programmed master assembly plan, and interact with their local environment and other components. Self-assembly is an autopoietic mechanism whose power, as a reusable engineering concept, lays in the fact that it is a distributed, not-necessarily synchronous, control mechanism for the bottom-up manufacture of complex systems. In nature, robustness, versatility, reversibility and mass transportation are important properties of these type of systems. The former comes from the fact that usually self-assembly systems are composed of a large number of parts that can be interchanged and replaced by one another in case one of them fails. Conversely, versatility is given by the possibilities of reconfiguring the way in which component parts relate to each other subject to, for example, attraction and repulsion forces.

Germán Terrazas, Graham Kendall and Natalio Krasnogor are at ASAP Group, School of Computer Science and IT, University of Nottingham, Nottingham, NG8 1BB, UK. Email: \{gzt, gxk, nxk\}@cs.nott.ac.uk

Marian Gheorghe is at Department of Computer Science, University of Sheffield, Sheffield, S1 4DP, UK. Email: M.Gheorghe@dcs.shef.ac.uk
Although major advances in the design of systems that exhibit self-assembly properties have been reported in the literature (e.g. [2] [3] [4]), much less has been said about the automated design of self-assembly. Continuing the research line of our previous findings [5], the intent of this paper is to report how self-assembly takes place as the complexity of the Wang tile model increases. As the models used here are richer than the ones used in [5] we also employ a more sophisticated fitness function based on Morphological Image Analyses. The following section introduces the theoretical background. Next, the evolutionary approach is presented. Finally, experiments with results analysis and conclusions follow.

\section{Wang Tiles and Self-Assembly}

Tiling theory has been widely used as a base model for many applications ranging from biology to computer science, e.g. DNA assembly [6], networks verification [7] and graphics rendering [8] [9] to name but a few.

This theory also serves as a link between computation and self-assembly [10]. A Wang tile system is defined as a set of square coloured-edge tiles embedded into the Euclidean plane. Tiles randomly move across the surface and in case of collision they either attach to one another or bounce off. As tiles stick to each other, they aggregate in complex structures and the specific emerging shapes are said to be self-assembled. What determines whether they stick or bounce is the colour of the colliding edges and the system's temperature.

$$
\begin{array}{r}
T=\left\{t \mid t=\left(x_{0}, x_{1}, x_{2}, x_{3}\right)\right\} \\
\text { where } \\
x_{0}, x_{1}, x_{2}, x_{3} \in \Sigma
\end{array}
$$

A formal definition of a set of Wang tiles $T$ is given in equation 1. The set $\Sigma$ contains a collection of labels encoding the "glue" types associated to each edge. Usually, it is also said that $x_{i}$ specifies a glue type represented by a colour code. Let $\tau$ be the "temperature" parameter modelling a specific kinetic energy associated to the tile set as defined in [11]. After colliding, two tiles $t_{i}, t_{j}$ will self-assemble by their edges $e_{i}, e_{j}$ if the glue types and strengths in those edges are compatible. The compatibility of different glue types is given by an interaction matrix which will specify for each pair of glue types/colours the strength of their bonding. If the bonding strength is greater than the temperature then the two colliding sides will bind. Hence, given a Wang tile system with a fixed $\tau$ and an interaction matrix, the goal of our approach is to design a 
TABLE I

WANG TILES SELF-ASSEMBLING.

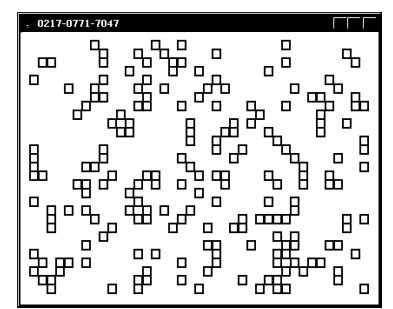

(a)

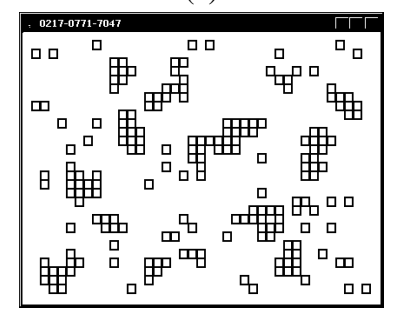

(e)

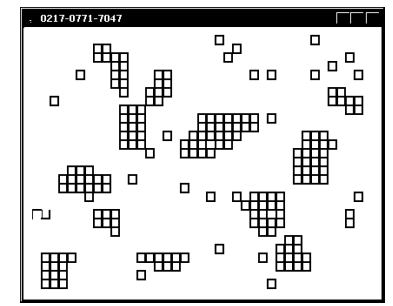

(i)

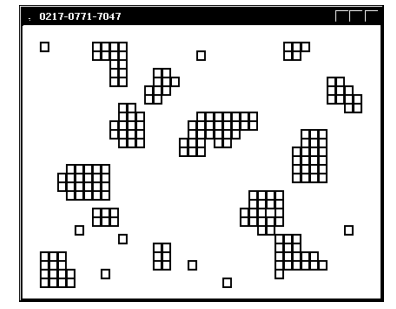

(m)

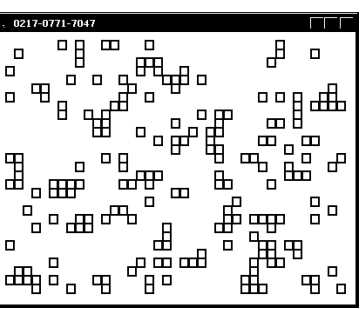

(b)

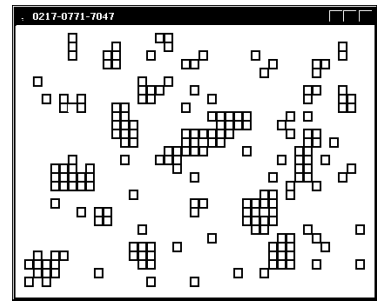

(f)

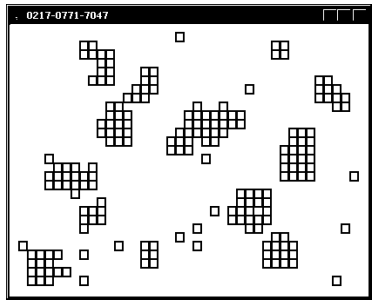

(j)

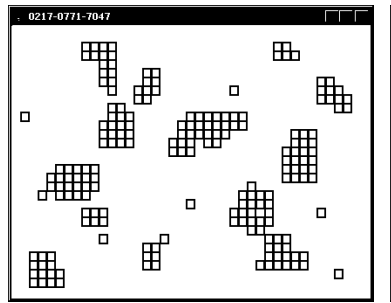

(n)

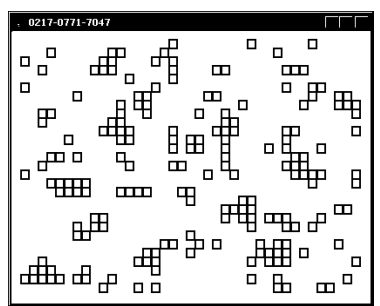

(c)

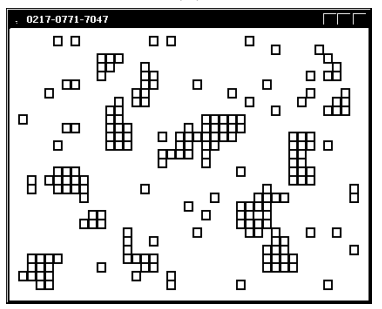

$(\mathrm{g})$

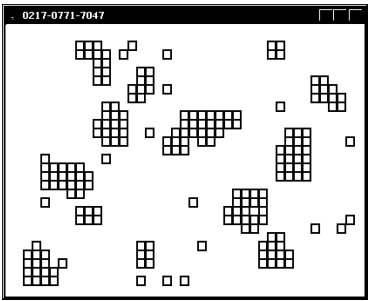

$(\mathrm{k})$

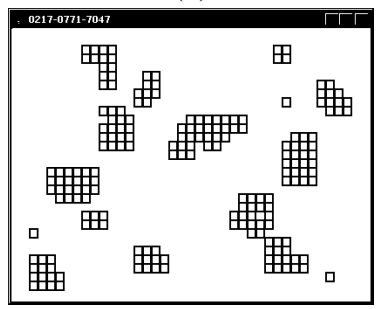

(o)

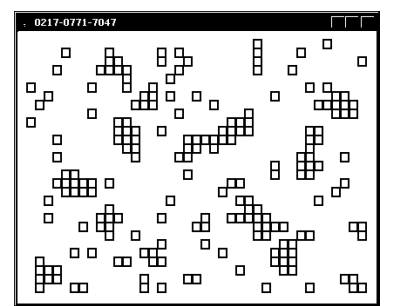

(d)

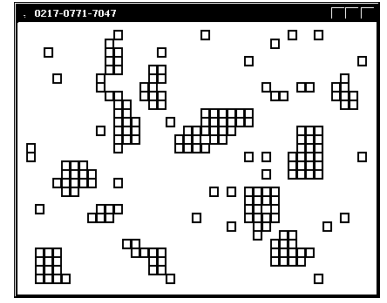

(h)

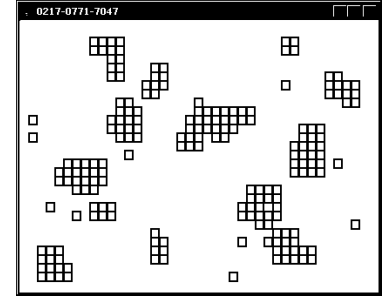

(1)

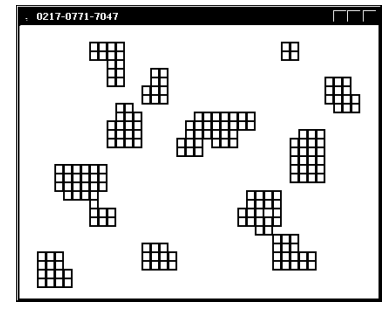

(p)

set of tiles capable to self-assemble into a specific (user defined) target shape. Previous works [6] [11] [12] in Wang tiles self-assembly have used as an interaction matrix a diagonal one. In this work, we further generalise that by removing the diagonalization constraint.

Table I shows a series of successive snapshots for a Wang tile system self-assembling into near square configurations. From figures (a) to (p), tiles wander across the lattice building conformations of different sizes.

\section{The Genetic Algorithm for Wang Tiles DESIGN}

The objective of the genetic algorithm is to search for the best set of tiles capable of building a target structure by means of self-assembly. As in our previous work [5], this target shape is defined as a two-dimensional square with size of ten by ten tiles.

\section{A. Representation and Initialisation}

The individuals of the population are defined as a collection of tile families. A family is a class of tiles that can be instantiated with several identical copies. These are arbitrarily initialised, i.e. each tile family is randomly assigned a colour to each of the four tiles sides. A formal 
definition for population and its variable-length individuals is shown below (equation 2).

$$
\begin{array}{r}
\text { Pop }=\left\{\text { Ind }_{1}, \text { Ind }_{2}, \ldots, \text { Ind }_{\delta}\right\} \\
\text { Ind }_{i}=\{\square, \square, \ldots, \underbrace{\square}_{T_{i}^{j}}\}
\end{array}
$$$$
\text { where } 1 \leq i \leq \delta, 1 \leq j \leq n_{i} \leq \varphi
$$

$$
T_{i}^{j} \text { defined as in equation } 1
$$

$\delta$ and $\varphi$ (population size and maximum number of families an individual contains) are fixed to all the experiments.

\section{B. Genetic Operators and Selection Procedures}

The genetic operators are one-point crossover and bitwise mutation. The crossover takes two individuals from the population $\operatorname{Ind}_{1}=\left\{T_{1}^{1}, T_{1}^{2}, \ldots, T_{1}^{n_{1}}\right\}$ and $\operatorname{Ind}_{2}=$ $\left\{T_{2}^{1}, T_{2}^{2}, \ldots, T_{2}^{n_{2}}\right\}$, selects the shorter individual and a random cutting point between tile families. Without loss of generality, if $n_{1}<n_{2}$ and the cutting point is 3 , then a possible offspring is $O=\left\{T_{1}^{1}, T_{1}^{2}, T_{1}^{3}, T_{2}^{4}, T_{2}^{5}, \ldots, T_{2}^{n_{2}}\right\}$. Parents are selected for mating using roulette-wheel selection, and the GA follows a generational scheme with single individual elitism. After a new generation is built, all its individuals are mutated with bit-wise mutation. More specifically, the mutation operator takes an individual and with a given low probability randomly changes one or more colours of the tile families in the individual.

\section{Evaluation}

The evaluation phase consists of two stages: tiles simulation and structure comparison.

1) Simulation: During the first stage, individuals are placed into a simulator composed by a two-dimensional square lattice. Essentially, for each tile family $T^{i}$ encoded by an individual an equal number of tile instances drawn from the family is placed into an empty position of the lattice. After that, tiles wander around sticking or bouncing until the simulation runs its course. There are four increasingly richer simulation environments:

Model 1. When two tiles reach adjacent locations, the glue function evaluates the interaction between the colours at touching edges using a symmetric matrix $M$ of $\alpha \times \alpha$ colours. If the resultant value is greater than the temperature $\tau$ (a strong interaction) both tiles self-assemble and remain in their locations; otherwise (a weak interaction) they move. For example, figure 1 shows a simulation with an interaction matrix of $\alpha=2$ and $\tau=4$. As it is depicted, the only way two tiles self-assemble is when black edges become adjacent as any other type of interaction is smaller than the temperature.

Model 2. In this case, if two tiles become adjacent, they are evaluated as in the previous model. The difference with Model 1 is the introduction of a probabilistic rather
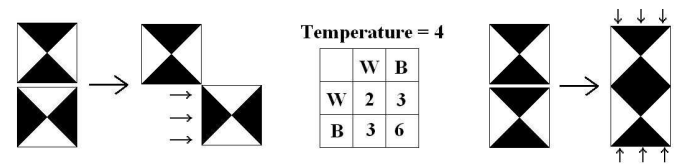

Fig. 1. Two-tiles interaction.

than deterministic stickiness criteria: $\rho$. For example, in the simulation shown in figure 2, tiles self-assemble as the sum of interactions is 6 . In this case, the central tile contributes with a strong interaction and remains in its position. In contrast, side tiles might still move as their interactions are weaker.

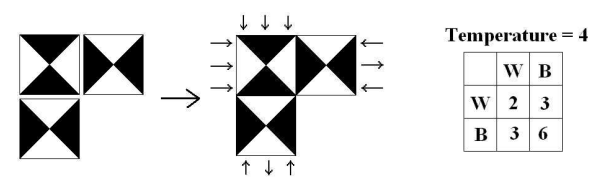

Fig. 2. Three-tiles interaction. The sum of interactions is 4 and the central tile keeps the side tiles assembled.

Equation 3 defines how $\rho$ is updated for a given tile $t$. At the beginning, the probability of being moved is set to one and the tile is free to move on the lattice. As a tile interacts with other tiles, $\rho$ either decreases when the tile becomes part of an aggregation, or rises when the tile leaves an aggregation. In any case, the calculation is performed in terms of the interaction value given by each edge of the tile, its neighbours and $\tau$.

$$
\begin{gathered}
\rho=\operatorname{Max}\left(0,1-\sum_{j=1}^{4} \operatorname{contact}\left(t, t_{j}\right) \cdot \frac{\text { strength }\left(t, t_{j}\right)}{\tau}\right) \\
t_{j}= \begin{cases}t_{1} & \text { is the northern neighbour of } t \\
t_{2} & \text { is the eastern neighbour of } t \\
t_{3} & \text { is the southern neighbour of } t \\
t_{4} & \text { is the western neighbour of } t\end{cases} \\
\text { contact }\left(t, t_{j}\right)= \begin{cases}1 & \text { if } t \text { is adjacent to } t_{j} \\
0 & \text { otherwise }\end{cases} \\
\text { strength }\left(t, t_{j}\right)= \begin{cases}M\left[t^{n}, t_{j}^{s}\right] & \text { if } j=1 \\
M\left[t^{e}, t_{j}^{w}\right] & \text { if } j=2 \\
M\left[t^{s}, t_{j}^{n}\right] & \text { if } j=3 \\
M\left[t^{w}, t_{j}^{e}\right] & \text { if } j=4\end{cases}
\end{gathered}
$$$$
t^{i} \text { is a tile edge, e.g. the north edge is } t^{n}
$$

When a tile contributing with a weak interaction moves, a different arrangement of tiles appears and $\rho$ is re-computed as interactions change. Figure 3 shows four alternatives in which the previous self-assembled structure could be modified. In the case of (a) and (d) the side tiles have moved away leaving a weak interaction among the remaining tiles and consequently disassembles the whole structure afterwards. Alternatively, in (b) and (c), the sum of interactions is either maintained or bigger than the original arrangement. Nevertheless, (b) and (c) show that with this model it is also possible to spin the whole structure. 


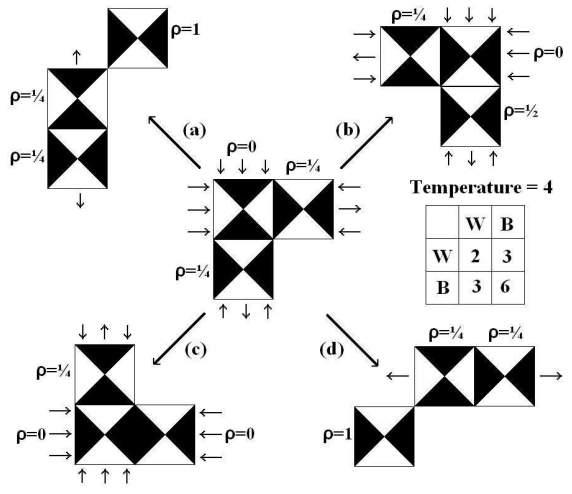

Fig. 3. Four alternatives in which a self-assembled structure composed by three tiles could be modified. Each tile re-calculates its $\rho$ as interactions with other tiles change.

To make the model richer we also allow tiles to rotate. That is, once a tile moves to a new position in the lattice, a random number is obtained. If it is in the interval $[-1,0]$ then the tile rotates to the left, else if it is in the interval $[0,1]$ it rotates to the right. So, considering the orientation of the grey edge depicted in figure 4 , a tile is able to rotate 90 degrees clockwise or counter clockwise.

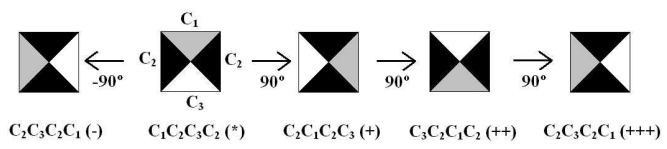

Fig. 4. Tile rotating clockwise or counter clockwise: $+(-)$ indicates the amount of clockwise (counter clockwise) rotations of a tile marked with *.

Hence, we define Model 3 and Model 4 as the combination of the two previous models with rotation, i.e. tiles without probabilistic motion plus rotation and tiles with probabilistic motion plus rotation. Thus, the complexity of the models increases from Model 1 to Model 4. Figure 5 shows a schematic representation of the four hierarchical models.

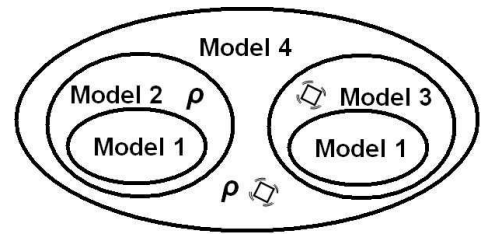

Fig. 5. Hierarchical scheme of the models.

2) Structure Comparison: After the simulation runs under Model i $(i \in[1,4])$ for $t$ time steps it is necessary to evaluate how close to the desired structure the self-assembling process is. In this context, two self-assembled structures are called close if they have a similar topology, arrangement and quantity of tiles. For this purpose, the Morphological Image Analysis method (MIA) was implemented as the core of the structure comparison stage. This method characterises the morphology of patterns in terms of geometrical and topological descriptors [13]. Research works belonging to the field of statistical physics [14], cosmology [15] [16], biology [17], seismology [18] and polymer sciences [19] have demonstrated that MIA is a robust approach to typify both 2-dimensional and 3-dimensional structures. This technique has its origins in the calculation of the so-called Minkowski functionals [20] which involves the computation of the area $(A)$, the perimeter $(U)$ and Euler characteristic $(\chi)$ of a given conformation. As an illustrative example, if we consider the 2-dimensional black pixelated drawing of figure 6 , then the area is the number of black pixels that make up the pattern, the perimeter is the number of lines making up the borders and the Euler is given by the connectivity of the pattern, i.e. the number of connected black pixels minus the number of completely enclosed white pixelated regions in the image.

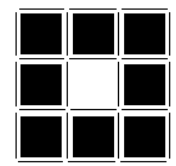

Fig. 6. Minkowski functionals for a black pixelated pattern with $A=8$, $U=16$ and $\chi=0$

However, given that rotated occurrences of a structure could appear, e.g. horizontal strips in vertical position, we enlarged the typification method calculating the radius of gyration $(R g)$ about the $\mathrm{x}$-axis. Thus, similarity among rotated structures are detected in terms of the moment of inertia and area around their centroidal axis.

All in all, the fitness function is defined as the morphological distance between the desired structure and those selfassembled by the tiles as shown in equation 4 .

$$
\text { Fitness }(\text { ind })=\sqrt{\Delta A^{2}+\Delta U^{2}+\Delta \chi^{2}+\Delta R g^{2}}
$$

$\Delta A=\frac{\sum_{j=1}^{n}\left|A_{T}-A_{j}\right|}{n}$ is the mean value of the distances between the area of the target structure and the areas of the resulting self-assembled structures.

$\Delta U=\frac{\sum_{j=1}^{n}\left|U_{T}-U_{j}\right|}{n}$ is the mean value of the distances between the perimeter of the target structure and the perimeters of the resulting self-assembled structures.

$\Delta \chi=\frac{\sum_{j=1}^{n}\left|\chi_{T}-\chi_{j}\right|}{n}$ is the mean value of the distances between the Euler of the target structure and the those of the resulting self-assembled structures.

$\Delta R g=\frac{\sum_{j=1}^{n}\left|R g_{T}-R g_{j}\right|}{n}$ is the mean value of the distances between the radius of gyration about $\mathrm{x}$-axis of the target structure and the radiuses of gyration of the resulting self-assembled structures. 
Figure 7 shows a schematic representation of the GA. At the beginning, population is initialised with sets of randomly created tile families. Then evaluation takes place combining the simulation and structure comparison stages. Finally, crossover and mutation follows.

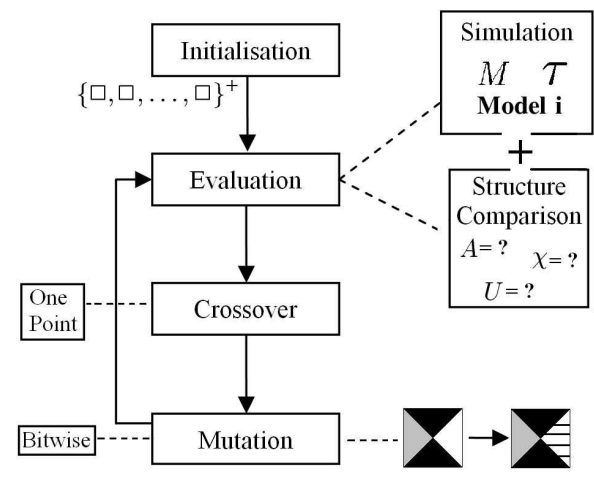

Fig. 7. Schematic representation of the GA.

\section{Methods And Results}

\section{A. Methods}

The four models defined in the previous section were used for the experiments. The simulation parameters and their values are shown in table II where $t$ stands for the length of the simulation, $\tau$ is the temperature of the system, $\mu$ is the number of glue strengths in the interaction matrix, LDim is the lattice dimension, $\alpha$ is the number of colours tinging the tile edges, $\lambda$ says whether or not rotation is set, and $\rho$ determines if the tile moves with probabilistic motion. Furthermore, table III shows the interaction matrix used by the glue function. Given two colours $C_{i}$ and $C_{j}$ the interaction strength between them is stated by $M\left[C_{i}, C_{j}\right]$.

TABLE II

TILE MOdEl PARAMETERS

\begin{tabular}{|c|c|c|c|c|c|c|}
\hline$t$ & $\tau$ & $\mu$ & LDim & $\alpha$ & $\lambda$ & $\rho$ \\
\hline 9000 & 4 & {$[0,9]$} & $800 \times 600$ & 10 & $\mathrm{Y} / \mathrm{N}$ & $\mathrm{Y} / \mathrm{N}$ \\
\hline
\end{tabular}

TABLE III

THE SYMMETRIC MATRIX RANDOMLY INITIALISED.

\begin{tabular}{c|cccccccccc|} 
& $C_{0}$ & $C_{1}$ & $C_{2}$ & $C_{3}$ & $C_{4}$ & $C_{5}$ & $C_{6}$ & $C_{7}$ & $C_{8}$ & $C_{9}$ \\
\hline$C_{0}$ & 7 & 2 & 7 & 7 & 3 & 0 & 0 & 1 & 7 & 1 \\
$C_{1}$ & 2 & 7 & 1 & 5 & 7 & 3 & 8 & 2 & 1 & 6 \\
$C_{2}$ & 7 & 1 & 6 & 4 & 8 & 9 & 2 & 2 & 5 & 1 \\
$C_{3}$ & 7 & 5 & 4 & 8 & 5 & 3 & 3 & 7 & 9 & 6 \\
$C_{4}$ & 3 & 7 & 8 & 5 & 8 & 7 & 5 & 0 & 3 & 9 \\
$C_{5}$ & 0 & 3 & 9 & 3 & 7 & 6 & 0 & 3 & 9 & 5 \\
$C_{6}$ & 0 & 8 & 2 & 3 & 5 & 0 & 1 & 8 & 8 & 5 \\
$C_{7}$ & 1 & 2 & 2 & 7 & 0 & 3 & 8 & 3 & 9 & 6 \\
$C_{8}$ & 7 & 1 & 5 & 9 & 3 & 9 & 8 & 9 & 7 & 0 \\
$C_{9}$ & 1 & 6 & 1 & 6 & 9 & 5 & 5 & 6 & 0 & 0 \\
\hline
\end{tabular}

For each model, five runs with three different values of mutation probability each were run. For all experiments we fixed the population size, the maximum length of the individuals, the crossover probability, the generations number and the shape dimension. Table IV shows the GA parameters and their values.

TABLE IV

Genetic Algorithm Parameters

\begin{tabular}{|c|c|c|c|c|c|}
\hline$\delta$ & $\mid$ Ind. $\mid$ & XProb & MProb & Gen & SDim \\
\hline 100 & 10 & 0.7 & $0.1 / 0.05 / 0.01$ & 300 & $10 \times 10$ \\
\hline
\end{tabular}

\section{B. Results}

The results are listed in table V. Under the $I d$ column, $M i X$ stands for the model type and number of experiment, e.g. $M 1 C$ is the third experiment using Model 1.

As it was expected, different models lead to different results. On the one hand, in Model 1 and Model 3 the performance of the individuals are quite similar regardless of rotation, i.e. fitness values do not go above 16 . On the other hand, if the model uses probabilistic motion, there is a remarkable difference on the evolved Wang tiles depending on whether or not rotation is set. For example, with Model 2 the GA found better solutions in terms of fitness than when using Model 4. Furthermore, it is also interesting to note that if tiles rotate the GA always reaches individuals composed with the same tile family as the best solutions.

TABLE V

RESULTS SUMMARY

\begin{tabular}{|c|c|c|c|}
\hline \multicolumn{4}{|c|}{ No Probabilistic Movement \& No Rotation } \\
\hline MProb & BestIndividual & Fitness & $I d$ \\
\hline 0.01 & {$[4483,0859,0869,0999,0969]$} & 15.319270 & M1A \\
\hline 0.1 & {$[5909,2449,5809]$} & 12.912152 & M1B \\
\hline 0.05 & {$[6622,9900]$} & 15.583095 & M1C \\
\hline \multicolumn{4}{|c|}{ Probabilistic Movement \& No Rotation } \\
\hline MProb & BestIndividual & Fitness & $I d$ \\
\hline 0.01 & {$[0217,0771,7047]$} & 35.57111 & M2A \\
\hline 0.1 & {$[7517,7410,7557]$} & 31.07997 & M2B \\
\hline 0.05 & {$[3550,6467]$} & 37.52435 & $\mathrm{M} 2 \mathrm{C}$ \\
\hline \multicolumn{4}{|c|}{ No Probabilistic Movement \& Rotation } \\
\hline MProb & BestIndividual & Fitness & $I d$ \\
\hline 0.01 & {$[9999,9088]$} & 13.719727 & M3A \\
\hline 0.1 & [8890, 9999] & 12.990824 & M3B \\
\hline 0.05 & {$[9999,6576,9999,9999]$} & 15.721738 & M3C \\
\hline \multicolumn{4}{|c|}{ Probabilistic Movement \& Rotation } \\
\hline MProb & BestIndividual & Fitness & $I d$ \\
\hline 0.01 & {$[7777,7777,7777,7777,7777,7777]$} & 27.830549 & M4A \\
\hline 0.1 & {$[7777,7777]$} & 27.004934 & M4B \\
\hline 0.05 & [7777] & 28.063046 & M4C \\
\hline
\end{tabular}

Beyond the conclusions involving the fitness value, it is also part of the analysis to discern how self-assembly is achieved by the evolved individuals. At this stage, we 
consider how many tiles are necessary for a particular individual - under the energy table shown above and with the specified temperature - to aggregate tiles into supra-structures for the different models tested.

1) Two-tiles self-assembly: With the best individuals of each experiment of table $\mathrm{V}$, we analysed all the combinations for assembling two tiles. That is, for every pair of tiles of an individual we analyse if there are combinations of glue types/colours with a strength greater than the temperature as it is depicted in figure 8.

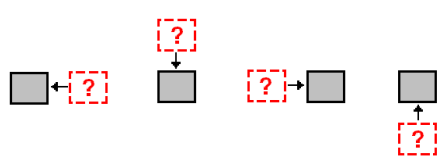

Fig. 8. Self-assembling conformations involving only two tiles.

Two-tiles assembly was found in some of the experiments using Model 1 and Model 3. For instance, the individuals of experiments M1A and M1B have just one tile, we call it seed tile, capable to stick with any other tile increasing in this way the chances to form small self-assembly aggregations at the beginning of the simulation. In addition, a similar behaviour is shown by the individuals of M3A, M3B and M3C. These analyses are shown in tables VI, VII, VIII and IX where each element of the first column, tiles, is the entry point in the table. The combination of N, S, E and W stands for which edges the selected tile is using for assembling, e.g. NS means that the tile assembles by north and south edges.

TABLE VI

TWO-TILE INTERACTION FOR EXPERIMENT M1A

\begin{tabular}{|c|c|c|c|c|c|}
\hline Tiles & 4483 & 0859 & 0869 & 0999 & 0969 \\
\hline $\mathbf{4 4 8 3}$ & EW & NSEW & NSEW & NSEW & NSEW \\
$\mathbf{0 8 5 9}$ & NSEW & - & - & - & - \\
$\mathbf{0 9 9 9}$ & NSEW & - & - & - & - \\
$\mathbf{0 8 6 9}$ & NSEW & - & - & - & - \\
\hline
\end{tabular}

TABLE VII

TWO-TILE INTERACTION FOR EXPERIMENT M1B

\begin{tabular}{|c|c|c|c|}
\hline Tiles & 5909 & 2449 & 5809 \\
\hline $\mathbf{5 9 0 9}$ & - & NSW & - \\
$\mathbf{2 4 4 9}$ & NSE & NSEW & NSE \\
$\mathbf{5 8 0 9}$ & - & NSW & - \\
\hline
\end{tabular}

Interestingly, in the experiments using Model 2 and Model 4, there is no possibility to generate two-tiles assemblies. That is, there are not two combinations of glue types/colours with a strength greater than the temperature in the system. Cooperation, thus, is an emergent feature of our system where more than two tiles are required to initiate self-assembly. Cooperation was recognised by Winfree and Rothemund in [12] as necessary for programmable selfassembly and it is a remarkable result that the evolutionary
TABLE VIII

TWO-TILE INTERACTION FOR EXPERIMENT M3 A AND M3B

\begin{tabular}{|l|c|c|c|c|c|}
\hline Tiles & 9999 & $9088(*)$ & $8908(+)$ & $8890(++)$ & $0889(+++)$ \\
\hline $\mathbf{9 9 9 9}$ & - & - & - & - & - \\
$\mathbf{9 0 8 8}(*)$ & - & EW & ES & ESW & SW \\
$\mathbf{8 9 0 8}(+)$ & - & NW & SN & SW & SWN \\
$\mathbf{8 8 9 0}(++)$ & - & EWN & EN & WE & WN \\
$\mathbf{0 8 8 9}(+++)$ & - & EN & ESN & ES & SN \\
\hline
\end{tabular}

TABLE IX

TWO-TILE INTERACTION FOR EXPERIMENT M3C

\begin{tabular}{|l|c|c|c|c|c|}
\hline Tiles & 9999 & $6576(*)$ & $6657(+)$ & $7665(++)$ & $5766(+++)$ \\
\hline $\mathbf{9 9 9 9}$ & - & NESW & NESW & NESW & NESW \\
$\mathbf{6 5 7 6}(*)$ & NESW & NS & S & E & W \\
$\mathbf{6 6 5 7}(+)$ & NESW & N & WE & W & S \\
$\mathbf{7 6 6 5}(++)$ & NESW & W & E & NS & N \\
$\mathbf{5 7 6 6}(+++)$ & NESW & E & N & S & WE \\
\hline
\end{tabular}

design of tiles presented here achieved precisely that (see below).

2) Three-tiles assembly: For those experiments where there is no possibility to generate two-tiles assembly we consider then the possible combinations of three tiles as shown in figure 9. Thus, the ?-tile interacts with the other two tiles attempting to attach to them and the site where tiles attach is defined as binding site. To make the analysis and characterisation of possible binding sites more tractable, we have divided the analysis into six binding site conformations accordingly to where the ?-tile could bind: the north-east, south-east, north-west, south-west, north (south) and east (west).

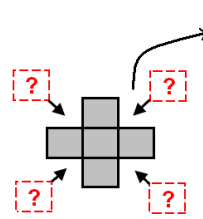

(a)

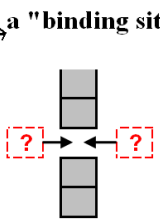

(b)

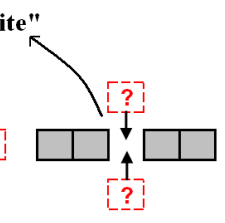

(c)
Fig. 9. The six binding site conformations.

Since more than one tile could potentially bind to a binding site conformation, for each of them we calculated its normalised average free energy (NAFE) [5]. Thus, equations $5,6,7$ and 8 calculate the NAFE for those binding site conformations where the ?-tile binds from north-east, southeast, north-west and south-west respectively (figure 9 (a)) whilst equations 9 and 10 compute the NAFE for those where the ?-tile binds from north (south) (figure 9 (b)) and east (west) (figure 9 (c)). In these equations, $G$ is the glue function where its arguments are tile edges, i.e. the north edge of the $i$-tile is $t_{i}^{n}$, and $|T|$ is the cardinality of the family.

In this way, it is possible to see which binding site conformation is in average more likely to participate in 
a three tiles assembly. We further partitioned the binding conformations in equivalence classes. Two binding conformations were deemed equivalent if their free energy were equal. The resulting partitions for experiments M1A, M1C, M2A, M2B, M2C, M4ABC - short for M4A, M4B and M4C - are shown in table XII.

$$
\begin{aligned}
& \frac{\sum_{?=1}^{|T|}\left(G\left(t_{i}^{e}, t_{?}^{w}\right)+G\left(t_{j}^{n}, t_{?}^{s}\right)\right)}{|T|} \\
& \frac{\sum_{?=1}^{|T|}\left(G\left(t_{i}^{e}, t_{?}^{w}\right)+G\left(t_{j}^{s}, t_{?}^{n}\right)\right)}{|T|} \\
& \frac{\sum_{?=1}^{|T|}\left(G\left(t_{i}^{w}, t_{?}^{e}\right)+G\left(t_{j}^{n}, t_{?}^{s}\right)\right)}{|T|} \\
& \frac{\sum_{?=1}^{|T|}\left(G\left(t_{i}^{w}, t_{?}^{e}\right)+G\left(t_{j}^{s}, t_{?}^{n}\right)\right)}{|T|} \\
& \frac{\sum_{?=1}^{|T|}\left(G\left(t_{i}^{s}, t_{?}^{n}\right)+G\left(t_{j}^{n}, t_{?}^{s}\right)\right)}{|T|} \\
& \frac{\sum_{?=1}^{|T|}\left(G\left(t_{i}^{e}, t_{?}^{w}\right)+G\left(t_{j}^{w}, t_{?}^{e}\right)\right)}{|T|}
\end{aligned}
$$

The analysed experiments show that the total amount of conformations provided by equivalence classes with NAFE lesser or equal than $\tau$ is bigger than the quantity provided by equivalence classes with greater NAFE. In other words, conformations with NAFE smaller than $\tau$ are highly likely to participate in the self-assembly process. Therefore, we have also analysed how many and which type of these binding sites were developed among individuals across evolution. Thus, an inspection over the resulting outcome in table $\mathrm{X}$ says that on the one hand, in those experiments using Model 1 few binding site conformations are employed. For instance, experiment M1A uses only one instance of a type and experiment $\mathrm{M} 1 \mathrm{C}$ one instance of each type. On the other

\begin{tabular}{|c|c|c|c|c|c|c|}
\hline & M1A & M1C & M2A & M2B & M2C & M4ABC \\
\hline$\square$ & & & & & & \\
\hline$\square$ & 1 & 1 & 15 & 27 & 1 & 1 \\
\hline$\square ? \square$ & - & 1 & 16 & 9 & 2 & 1 \\
\hline$\square ?$ & - & 1 & 16 & 15 & 3 & 1 \\
\hline ?1 & - & 1 & 17 & 15 & 3 & 1 \\
\hline$\square$ & - & 1 & 14 & 15 & 3 & 1 \\
\hline 10 & - & 1 & 19 & 15 & 3 & 1 \\
\hline
\end{tabular}
hand, for Model 2 and Model 4, it turns out that 97 instances of binding site conformations are employed by M2A, 96 by $\mathrm{M} 2 \mathrm{~B}, 15$ by $\mathrm{M} 2 \mathrm{C}$ and 6 by experiment M4ABC.

TABLE $X$

THREE-TILES ASSEMBLY ANALYSIS

3) Four-tiles assembly and beyond: Further analysis shows that there are four-tiles and five-tiles cooperative behaviour that boost self-assembly as it is shown in figure 10 . These type of interactions were found in some individuals of Model 1 and Model 2. The amount of conformations for experiments M1C and M2A are listed in table XI. For instance, the former uses two cases of four-tiles cooperation and one involving five tiles whilst the latter employs in total 26 instances in case of four-tiles cooperation.

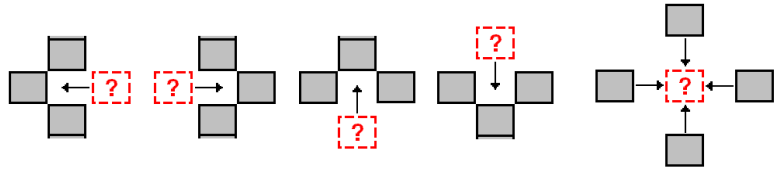

Fig. 10. Self-assembling conformations involving four and five tiles.

TABLE XI

FouR-TILES AND FIVE-TILES ASSEMBLy ANALYSIS

\begin{tabular}{|l|c|c|c|c|c|}
\hline & $\square ?$ & $\square ? \square$ & $\square ?$ & $?$ & $\square ?$ \\
\hline M1C & - & 1 & 1 & - & 1 \\
M2A & 7 & 5 & 9 & 5 & - \\
\hline
\end{tabular}

As an overall conclusion, it is interesting to note that the less complex the tile model is, the smaller are the resulting aggregations as two-tiles interactions become not only common but also frequent building blocks. In contrast, experiments using tiles assisted with probabilistic motion result in bigger aggregations due to the usage of three-tiles, four-tiles and five-tiles cooperativeness. In other words, probabilistic motion necessitates intermediate building blocks, called generalised secondary structures in [21], for adequate self-assembly to occur. Nevertheless, if we measure the length of the individuals listed in table $\mathrm{V}$ we can conclude that when tiles are allowed to rotate the number of families to instantiate is smaller than when tiles do not rotate. That is, the type of tiles needed to create the target shape is minimised. Thus, it seems that a conservation of complexity principle is operating whereby the richer the environment the simpler the individuals and, conversely, the simpler the environment the more complex the individuals.

\section{Conclusions}

This paper reported on an evolutionary approach for the automated design of self-assembling tiles. The assembly model we used is that of Wang tiles extended with probabilistic motion and rotation. Thus, substantially extending our previous work, we showed a principled analysis of the evolved tiles which explains how selfassembly is achieved. In future work we will consider the self-assembling of a variety of shapes and the possible extension of the presented models. Related works we have been working on can be find in [22], [23] and [24].

\section{Acknowledgements}

N. Krasnogor would like to acknowledge EPSRC for funding project EP/D021847/1 and EP/E017215/1. 


\section{REFERENCES}

[1] L. Li, N. Krasnogor and J. Garibaldi, Automated self-assembly program ming paradigm: Initial investigations, In Proceedings of the Third IEEE International Workshop on Engineering of Autonomic \& Autonomous Systems, Potsdam, Germany, pp. 25-34, 2006

[2] P.W.K. Rothemund, Using Lateral Capillary Forces to Compute by SelfAssembly, Proceedings of the National Academy of Science, USA, vol. 47, pp. 984-989, 2000

[3] G.M. Whitesides and B. Grzybowski, Self-Assembly at all Scales, Science, vol. 295, pp. 2418-2421, 2002.

[4] G.M. Whiteside and M. Boncheva, Beyond Molecules: Self-Assembly of Mesoscopic and Macroscopic Components, Proceedings of the National Academy of Science (PNAS), vol. 99, pp. 4769-4774, 2002.

[5] G. Terrazas, N. Krasnogor, G. Kendall and M. Gheorghe, Automated Tile Design for Self-Assembly Conformations, Proceedings of the 2005 IEEE Congress on Evolutionary Computation, Edinburgh, U.K., vol. 2, pp. 1808-1814, Sep. 2005.

[6] T.H. LaBean, E. Winfree, J.H. Reif, Experimental Progress in Computation by Self-Assembly of DNA Tilings, DNA Based Computers V: DIMACS Workshop - American Mathematical Society, 1999.

[7] V.R. Syrotiuk, C.J. Colbourn and J. Pachl, Wang Tiling and Distributed Verification on Anonymous Torus Networks, Theory Computers Systems, vol. 30, pp. 145-163, 1997.

[8] M. Alsweis, O. Deussen, Wang-Tiles for the Simulation and Visualization of Plant Competition, 24th Computer Graphics International Conference 2006, Springer-Verlag LNCS 4035, pp. 1-11, 2006.

[9] M.F. Cohen, J. Shade, S. Hiller and Oliver Deussen, Wang Tiles for image and texture generation, SIGGRAPH '03: ACM SIGGRAPH 2003 Papers, ACM Press, pp. 287-294, 2003.

[10] H. Wang, Proving Theorems by Pattern Recognition, Bell Systems Technical Journal, vol. 40, pp. 1-42, 1961.

[11] L. Adleman, Q. Cheng, A. Goel, M. Huang, D. Kempe, P. Moisset de Espanes and P.W.K. Rothemund, Combinatorial Optimization Problems in Self-Assembly, Proceedings of the Annual ACM Symposium on Theory of Computing(STOC), ACM Press, 2002.

[12] P.W.K. Rothemund and E. Winfree, The Program-Size Complexity of Self-Assembled Squares, Proceedings of STOC, 2000.

[13] K. Michielsen and H. De Raedt, Morphological image analysis, Computer Physics Communications, vol. 1, pp. 94-103, 2000.

[14] J.S. Kole, K. Michielsen and H. De Raedt Morphological Image Analysis of Quantum Motion in Billiards, Physical Review, v. E63, 2001

[15] J. Schmalzing, M. Kerscher and T. Buchert Minkowski Functionals in Cosmology, Proceedings of Int. School of Physics Enrico Fermi, Varenna, 1995.

[16] A.V. Mordvinov, I.I. Salakhutdinova, L.A. Plyusnina, N.G. Marenko and L.M. Karimova The Topology of Background Magnetic Fields and Solar Flare Activity, Solar Physics Journal, v. 211, pp. 241-253, 2002.

[17] M.S. Barbosa, L. da Fontoura Costa and E. de Sousa Bernardes, Neuromorphometric characterization with shape functionals, Physical Review E, v. 67, 2003

[18] L. Karimova, N. Makarenko, Analysis of spatiotemporal data by Minkowski functionals, EGS - AGU - EUG Joint Assembly, April 2003 France.

[19] K. Michielsen, H. De Raedt and J.G.E.M. Fraaije Morphological Characterization of Spatial Patterns, Prog. Theor. Phys. Suppl., v. 138, pp. 543-548, 2000.

[20] D. Stoyan, W.S. Kendall and J. Mecke, Stochastic Geometry and its Applications, Akademie Verlag, Berlin, 1989.

[21] N. Krasnogor, G. Terrazas, D.A. Pelta and G. Ochoa, A Criticial View of the Evolutionary Design of Self-Assembling Systems, Proceedings of the 7th International Conference on Artificial Evolution, Special track on Self-Assembly, October 2005, Lille, France, 2005.

[22] P. Siepmann, G. Terrazas and N. Krasnogor, Evolutionary Design for the Behaviour of Cellular Automaton-Based Complex Systems, ACDM'06 Adaptive Computing in Design and Manufacture, pp. 199208, April 2006, Bristol, UK.

[23] G. Terrazas, P. Siepmann, G. Kendall and N. Krasnogor, An Evolutionary Methodology for the Automated Design of Cellular Automatonbased Complex Systems, Journal of Cellular Automata, v. 2, pp. 77-102, 2007.

[24] P. Siepmann, C.P. Martin, I. Vancea, P.J. Moriarty and N. Krasnogor, $A$ Genetic Algorithm Approach to Probing the Evolution of Self-Organised Nanostructured Systems, to appear in Nano Letters.
TABLE XII

NAFE RESUlTS For EXPERIMENTS M1A, M1C, M2A, M2B, M2C, $\mathrm{M} 4 \mathrm{ABC}$

\begin{tabular}{|c|c|c|c|c|c|}
\hline \multicolumn{6}{|c|}{ Individual 4483-0859-0869-0999-0969 (M1A) } \\
\hline $\mathrm{EqC}$ & NAFE & Qty & $\mathrm{EqC}$ & NAFE & Qty \\
\hline $\mathrm{i}$ & 7.0 & 2 & xvii & 7.4 & 2 \\
\hline ii & 8.6 & 4 & xviii & 3.6 & 8 \\
\hline iii & 3.2 & 6 & xix & 15.2 & 1 \\
\hline iv & 2.6 & 10 & $\mathrm{xx}$ & 12.8 & 1 \\
\hline $\mathrm{v}$ & 6.8 & 2 & $\mathrm{xxi}$ & 13.2 & 1 \\
\hline vi & 8.0 & 8 & xxii & 3.8 & 2 \\
\hline vii & 3.4 & 24 & xxiii & 8.4 & 2 \\
\hline viii & 7.8 & 4 & xxiv & 10.0 & 4 \\
\hline ix & 7.6 & 6 & $\mathrm{XXV}$ & 7.2 & 1 \\
\hline $\mathrm{x}$ & 4.4 & 6 & xxvi & 3.0 & 12 \\
\hline xi & 8.8 & 2 & xxvii & 9.8 & 4 \\
\hline xii & 9.2 & 2 & xxviii & 12.0 & 1 \\
\hline xiii & 2.2 & 4 & xxix & 14.4 & 1 \\
\hline xiv & 9.6 & 2 & $\mathrm{xxx}$ & 2.8 & 20 \\
\hline$x V$ & 8.2 & 2 & xxxi & 10.8 & 1 \\
\hline xvi & 14.0 & 1 & xxxii & 4.2 & 4 \\
\hline \multicolumn{6}{|c|}{ Individual 6622-9900 (M1C) } \\
\hline $\mathrm{EqC}$ & NAFE & Qty & $\mathrm{EqC}$ & NAFE & Qty \\
\hline $\mathrm{i}$ & 2.0 & 6 & iv & 2.5 & 8 \\
\hline ii & 1.50 & 8 & $\mathrm{v}$ & 1.0 & 1 \\
\hline iii & 3.0 & 1 & & & \\
\hline \multicolumn{6}{|c|}{ Individual 0217-0771-7047 (M2A) } \\
\hline $\mathrm{EqC}$ & NAFE & Qty & $\mathrm{EqC}$ & NAFE & Qty \\
\hline $\mathrm{i}$ & 3.0 & 3 & iv & 4.66 & 6 \\
\hline ii & 3.33 & 12 & $\mathrm{v}$ & 4.33 & 3 \\
\hline iii & 3.66 & 18 & vi & 4.0 & 12 \\
\hline \multicolumn{6}{|c|}{ Individual 7517-7410-7557 (M2B) } \\
\hline $\mathrm{EqC}$ & NAFE & Qty & $\mathrm{EqC}$ & NAFE & Qty \\
\hline $\mathrm{i}$ & 2.0 & 1 & $\mathrm{v}$ & 3.0 & 8 \\
\hline ii & 5.0 & 4 & vi & 4.33 & 18 \\
\hline iii & 5.33 & 3 & vii & 4.0 & 14 \\
\hline iv & 3.33 & 6 & & & \\
\hline \multicolumn{6}{|c|}{ Individual 3550-6467 (M2C) } \\
\hline $\mathrm{EqC}$ & NAFE & Qty & $\mathrm{EqC}$ & NAFE & Qty \\
\hline $\mathrm{i}$ & 2.0 & 5 & iv & 3.0 & 8 \\
\hline ii & 3.5 & 4 & $\mathrm{v}$ & 2.5 & 1 \\
\hline iii & 5.0 & 1 & vi & 4.5 & 5 \\
\hline \multicolumn{6}{|c|}{ Individual 7777 (M4ABC) } \\
\hline \multicolumn{2}{|c|}{$\mathrm{EqC}$} & \multicolumn{2}{|c|}{ NAFE } & \multicolumn{2}{|c|}{ Qty } \\
\hline \multicolumn{2}{|c|}{$\mathrm{i}$} & \multicolumn{2}{|c|}{6.0} & \multicolumn{2}{|c|}{6} \\
\hline
\end{tabular}

